Instructions for authors, subscriptions and further details:

\title{
La Formación Ciudadana en la Educación Secundaria Obligatoria en Cataluña. Análisis del concepto de Ciudadanía en el Currículum
}

Isidora Sáez-Rosenkranz ${ }^{1}$

Ilaria Bellatti ${ }^{1}$

Dolors Mayoral ${ }^{2}$

1) University of Barcelona, Spain

2) University of Lleida, Spain

Date of publication: February $25^{\text {th }}, 2017$

Edition period: February 2017-June 2017

To cite this article: Sáez-Rosenkranz, I., Bellati, I., Mayoral, D. (2017). La Formación Ciudadana en la Educación Secundaria Obligatoria en Cataluña. Análisis del concepto de Ciudadanía en el Currículum. International Journal of Sociology of Education, 6(1), 110-131. doi: 10.17583/rise.2017.2471

To link this article: http://dx.doi.org/10.17583/rise.2017.2471

PLEASE SCROLL DOWN FOR ARTICLE

The terms and conditions of use are related to the Open Journal System and to Creative Commons Attribution License (CC-BY) 


\section{Citizenship Education Secondary Compulsory Education in Catalonia. An Analysis on Citizenship's Concept within the Curriculum}

Isidora Sáez-Rosenkranz

University of Barcelona
Ilaria Bellatti

University of Barcelona
Dolors Mayoral

University of Lleida

(Received: 29 December 2016; Accepted: 11 January 2017; Published: 25 February 2017)

\section{Abstract}

This paper corresponds to the first stage of a major research that names this monograph. This text analyzes the conceptions on citizenship into the curricular concretions that the Catalonian Autonomic Community made of the official educational syllabus for the secondary compulsory education of the former educational law (LOE). Its aim is to verify the linking between curricular statements and teaching directions. Documental analysis is used, specifically content and discursive analysis by emphasizing into the meanings, implicit and explicit, on citizenship stated curricular orientations. As well it discusses the possible teaching consequences of these relations. One of the main results is that there is a gasp between what is stated into the prescriptive sections and teaching orientations, which are oriented to promote by teachers' citizenship competences on their students.

Keywords: citizenship, curriculum, citizenship education, secondary compulsory education. 


\section{La Formación Ciudadana en la Educación Secundaria Obligatoria en Cataluña. Análisis del concepto de Ciudadanía en el Currículum}

Isidora Sáez-Rosenkranz

University of Barcelona
Ilaria Bellatti

University of Barcelona
Dolors Mayoral

University of Lleida

(Recibido: 29 Diciembre 2016; Aceptado: 11 Enero 2017; Publicado: 25 Febrero 2017)

\section{Resumen}

El presente artículo constituye la primera fase de la investigación desarrollada y que da nombre a este monográfico. En él se analiza cómo se concibe la ciudadanía en las concreciones curriculares de la Comunidad Autónoma de Catalunya del plan de estudio oficial dirigida a la Educación Secundaria Obligatoria de la anterior ley educativa (LOE), para comprobar hasta qué punto este relato es coherente con las directrices que el marco técnico de la educación obligatoria presenta a los docentes. Para ello, se emplea el análisis documental, específicamente el análisis de contenido y discursivo, poniendo énfasis en los significados que las directrices curriculares atribuyen de forma implícita y explicita al concepto de ciudadanía, así como sus posibles consecuencias en el planteamiento didáctico. Uno de los principales resultados es la existencia de una brecha entre lo declarado en las partes prescriptivas y normativas del currículo, casi a nivel de principios, y las secciones didácticas. Éstas últimas se presentan bajo forma de orientaciones dirigidas al profesorado para la formación ciudadana de los alumnos de la enseñanza secundaria obligatoria.

Palabras clave: ciudadanía, currículum, formación ciudadana, enseñanza secundaria obligatoria (ESO) 


\section{Sáez-Rosenkranz, Bellatti \& Mayoral-Ciudadanía}

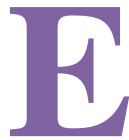

1 análisis de los conceptos sociopolíticos de los currículos de Historia, Educación para la Ciudadanía y Educación Ética, trata de resolver el primer objetivo de una investigación más amplia ${ }^{1}$. Esta tuvo por objeto contribuir al conocimiento sobre qué y cómo se abordan los contenidos sociopolíticos desde las materias de Ciencias Sociales. De esta manera se apuntó a comprender el impacto que tiene la escuela en las percepciones y valoraciones del alumnado sobre la convivencia intercultural y la participación ciudadana. Así, en la primera fase del proyecto se establecía: Analizar los contenidos y las estrategias didácticas empleadas en las aulas de Ciencias Sociales de la Educación Secundaria Obligatoria (ESO), empleadas para trabajar sobre las siguientes temáticas: a) la interculturalidad; b) convivencia y diálogo entre culturas; c) el funcionamiento del aparato democrático en sociedades plurales; d) los instrumentos de participación ciudadana existentes en la sociedad actual.

Para lograr este objetivo, se desarrolló un análisis de contenido de los apartados prescriptivos y didácticos de la concreción curricular de todas las etapas de la Educación Secundaria Obligatoria (ESO) de Cataluña (Generalitat de Catalunya, 2007b) que materializó la adaptación de la Ley Orgánica de Educación 2/2006 del Estado español (LOE) ${ }^{2}$. La concreción curricular, al igual que la ley, estaba estructurada en torno a competencias, las cuales son el eje del proceso educativo.

En el currículum de la ESO hay dos tipos de competencias: las transversales y las específicas. Las primeras, concebidas como la base del desarrollo personal y de construcción del conocimiento, incluyen la competencia comunicativa, lingüística y audiovisual; la artística y cultural; el tratamiento de la información y competencia digital; la competencia matemática; la de aprender a aprender y la de autonomía e iniciativa personal. Las segundas orientadas a convivir y habitar en el mundo, engloban las competencias en el conocimiento e interacción con el mundo físico y la competencia social y ciudadana.

Se aspiraba a que la educación ciudadana fuese un ámbito trabajado a lo largo de toda la ESO (Generalitat de Catalunya, 2007a) aunque se profundizaba en tercero y cuarto año del recorrido escolar con tópicos específicos. Así, en tercero se abordaba la educación para la ciudadanía y los derechos humanos y en cuarto educación ético-cívica. En España se decidió 
integrar la asignatura al currículum oficial según las disposiciones empezadas por el Consejo Europeo a finales de los años noventa, en todos los países miembros.

La alta injerencia que presentó la formación ciudadana en el currículum, tanto por su presencia como una competencia a desarrollar a lo largo de toda la etapa, como en una asignatura en la mitad de los cursos de la ESO, invitan a indagar en el sentido y significado que tiene este concepto dentro del currículum.

De forma particular, el interés por indagar cómo se concibe la ciudadanía en el currículum radica en dos aspectos centrales. En primer lugar, siguiendo a César Coll, "preside las actividades las actividades educativas escolares, precisa sus intenciones y proporciona guías de acción adecuadas y útiles para los profesores que tienen la responsabilidad directa de su ejecución" (1987, p. 31). En segundo lugar, porque juega un papel destacado en la configuración de significados y valores, convirtiéndose en un instrumento educativo a la vez que político. De esta manera, el currículum tiene una dimensión simbólica, didáctica y pedagógica (Gimeno Sacristán, 1988; Stenhouse, 1997). El currículm, a pesar de presidir los aprendizajes de aula, como bien señala Torres Santomé (1991), el colectivo docente acostumbra a aplicar sin mediar procesos reflexivos.

De esta manera, el presente artículo tiene dos objetivos centrales. Por un lado se pretende analizar cómo se concibe discursivamente la ciudadanía en el currículum de ESO de Cataluña; y por otro, revisar las directrices a los docentes sobre la formación en ciudadanía para determinar la coherencia entre lo discursivo de las orientaciones pedagógicas y los contenidos de la programación didáctica.

\section{Ciudadanía, Formación Ciudadana y Ciencias Sociales}

La educación institucional y especialmente la escuela como su principal materialidad tienen por fin último la formación de sujetos con determinados aprendizajes, valores y capacidades para desenvolverse en la vida social. Esta visión política implica que el objetivo de los sistemas educativos es formar ciudadanos (Cox, Bascopé, Castillo, Miranda, \& Bonhomme, 2014; Print, 2003).

Lizcano Fernández (2012) señaló que el concepto de ciudadanía tiene dos 


\section{Sáez-Rosenkranz, Bellatti \& Mayoral-Ciudadanía}

acepciones. Por un lado una concepción amplia en el sentido de pertenencia a una comunidad, y por otra, una acepción restringida ligada a "la enunciación de las características que deben tener los habitantes de una colectividad para poder ser considerados como ciudadanos" (p.3). En cualquiera de los dos casos, una primera distinción es que la ciudadanía enviste una connotación política en su orientación de lo político -como algo inherente a las relaciones humanas- y de la política -establecimiento del orden y organización de la coexistencia humana (Arendt, 1997; Mouffe, 1999).

Lizcano Fernández (2012) en su balance sobre las definiciones de ciudadano, acogiéndose a la dimensión restringida del término, estableció que la literatura lo define de dos maneras, una jurídico-político y otra éticopolítico. En la primera conceptualización, las nociones clave son los derechos y deberes políticos, mientras que en la segunda se incorporan también la idea de vida pública con valores cívicos. Aquí el autor consideró que el enfoque ético-político

"no tiene un interés cognitivo, sino que además pretende servir de base para proyectar y llevar a cabo acciones concretas encaminadas a la construcción de ciudadanía; es decir, a la expansión y fortalecimiento de buenos ciudadanos, tarea que debe involucrar a quienes todavía no lo son pero lo serán con el paso del tiempo: los niños" (Lizcano Fernández, 2012, p. 15).

Chantal Mouffe (1999) sostuvo que en el desarrollo de la democracia, la presencia de valores ético-políticos son fundamentales. La ciudadanía concibe al ciudadano como depositario de derechos, deberes y valores cívicos para participar en la vida democrática. Esto es lo que Print (2003) denominó ciudadanos democráticos.

Para actuar políticamente, los ciudadanos requieren de una identidad política común que, al margen de sus diferencias individuales, les permitan operar mediante la identificación con la res publica (Mouffe, 1999). Sara Kries (2006) señaló que la ciudadanía desde su origen está relacionada con la preocupación de la cosa pública, pero en sociedades democráticas, o al menos en el ideal de sociedades democráticas, debiese considerar a todos los ciudadanos en condiciones de igualdad (Cox et al., 2014; Mouffe, 1999). 
Mas, la democracia, como retoman Cox y otros (2014), va contra la naturaleza humana y por tanto, la ciudadanía no es inherente sino que se hace. Es una construcción donde la educación y la escuela juega un papel central.

La construcción de ciudadanía en la escuela ha estado tradicionalmente asociada a su dimensión cívica (Cristol, Michell, \& Gimbert, 2010) quedando como secundario el ámbito civil. Esto implica que, a pesar de que la formación ciudadana debiese apuntar a promover diversos tipos de aprendizajes orientados al desenvolvimiento de los sujetos sociales (Print, 2003), en aula se han promovido aspectos propios de contenidos institucionales. Sin embargo, en los últimos años se ha observado una creciente ampliación de la formación ciudadana hacia otras dimensiones del mundo colectivo (Cristol et al., 2010)

En la enseñanza de las ciencias sociales la formación ciudadana ha tenido un lugar secundario (Minte, Orellana, \& Tello, 2013) y su promoción ha sido escasa (López Atxurra \& de la Caba Collado, 2012). Las ciencias sociales son un ámbito idóneo para promover aprendizajes que permitan que el estudiante se desempeñe en sociedad (Prats, 2010) tales como los debates en torno a temas sensibles, la participación y toma de decisiones, resolución de problemas y rigor metodológico (López Facal \& Valls Montés, 2012). A su vez, las ciencias sociales propenden la formación ciudadana cuando se favorece el trabajo colaborativo, actividades que promueven la metacognición y procesos cognitivos complejos (López Atxurra \& de la Caba Collado, 2012).

Tal y como se la concibe actualmente, la formación ciudadana debiese promover la adquisición de conocimientos, actitudes, valores que permitan un comportamiento adecuado en democracia (Print, 2003). Asimismo, el conocimiento de la historia y los procedimientos propios de la investigación social debiesen ser considerados (López Facal \& Valls Montés, 2012) por entregar las bases cognitivas para la comprensión de la vida en sociedad. De esta manera, una educación para la ciudadanía tendría que poner el énfasis en el estudiante, empleando metodologías activas de aprendizaje. En palabras de Murray Print, "deberán enseñarse utilizando estrategias pedagógicas que pongan el acento sobre unos papeles activos, participativos, conceptualmente retadores, críticos y cooperativos para los estudiantes" (2003, p. 14). 


\section{Sáez-Rosenkranz, Bellatti \& Mayoral-Ciudadanía}

\section{Metodología}

Metodológicamente esta parte de la investigación se realizó utilizando el análisis de contenido en el currículum de ciencias sociales y educación para la ciudadanía de la Educación Secundaria Obligatoria de Cataluña vigente hasta el curso 2014/2015. Se escogió este tipo de análisis porque su objetivo central es indagar en los significados de las ideas expresadas en el texto (López Noguero, 2002), puesto que profundiza en los aspectos simbólicos de los mensajes (Krippendorff, 1990). Esta forma de abordar el currículum permite entender las características bajo las cuales se concibe institucionalmente la formación ciudadana, y cómo son las directrices que se entrega al profesorado en Cataluña.

Este método es flexible a la hora de procesar los datos, ya sea desde una perspectiva cualitativa o cuantitativa. De esta manera, se realizó un análisis de frecuencias que derivó en la revisión de las relaciones intertextuales de los conceptos. La premisa de que el texto es fruto de procesos singulares de comunicación (Piñuel Raigada, 2002) y en tanto tal, puede presentar contenidos ya sea explícitos u ocultos dentro de un contexto mayor, favoreció la posibilidad de indagar en lo dicho y lo no dicho. Así, el currículum de Ciencias Sociales de ESO de Cataluña se concibió como producto de lo que la Comunidad Autónoma de Cataluña emite al profesorado para que guíe su acción áulica bajo determinados mensajes, que el análisis de contenidos nos permitió develar.

Siguiendo la síntesis metodológica planteada por Piñuel Raigada (2002), el análisis de contenido desarrollado se resume en la siguiente tabla.

Tabla 1

Pasos del análisis de contenido

\begin{tabular}{|c|c|}
\hline Pasos & Aplicación a la investigación \\
\hline $\begin{array}{l}\text { Selección de la comunicación } \\
\text { estudiada }\end{array}$ & Currículum de Ciencias Sociales, ESO, Cataluña \\
\hline Selección de las categorías & Conceptos sociopolíticos \\
\hline Selección de las unidades de análisis & Tipología del concepto y conceptos asociados \\
\hline Sistema de recuento y medida & $\begin{array}{l}\text { Frecuencias y porcentajes, localización dentro del } \\
\text { documento. }\end{array}$ \\
\hline (Piñuel Raigada, 2002) & \\
\hline
\end{tabular}


Se escogió el concepto de ciudadanía y sus palabras asociadas (ciudadano y ciudadana), en tanto, es la idea clave de la formación en este ámbito. Gracias a su análisis contextual fue posible apreciar cómo esta es concebida y a con qué orientaciones pedagógicas está vinculada.

El procedimiento de análisis de este concepto se realizó de la siguiente manera ${ }^{3}$ :

1. Lectura del Currículum de ESO, en la cual se planteó una primera recogida emergente de conceptos sociopolíticos a analizar.

2. Delimitación de los apartados curriculares a analizar, en la cual se decidió trabajar con la asignatura de Ciencias Sociales y Educación para la ciudadanía de la Educación Secundaria Obligatoria de Cataluña.

3. Búsqueda de conceptos sociopolíticos, entre ellos ciudadanía, mediante el software Weft QDA en un margen de texto de 100 caracteres.

4. Individualización del conceptos y clasificación según el apartado del Currículum en el que aparece:

a. Ciencias Sociales: Introducción, Primer, Segundo, Tercer y Cuarto Curso.

b. Educación para la Ciudadanía: Introducción, Tercer y Cuarto Curso.

5. Análisis de los conceptos:

a. Clasificación de la palabra.

b. Verbos asociados a la palabra.

c. Conceptos asociados a la palabra.

6. Análisis de las directrices pedagógicas para la formación de la ciudadanía.

7. Interpretación del concepto a partir de la triangulación de los resultados anteriores.

\section{Concepto de "Ciudadanía" en Contexto del Currículum}

De la recogida emergente de conceptos sociopolíticos, encontramos 71 con frecuencias que van desde un máximo de ciento veintiocho veces hasta una vez (Gráfico 1). La palabra que mayor cantidad de veces aparece es 


\section{Sáez-Rosenkranz, Bellatti \& Mayoral-Ciudadanía}

"Mundo", seguido de "Cultura", "Sociedad" y "Ciudadanía".

Estos primeros cuatro conceptos, son generales mientras que, la mayoría de los que les suceden en frecuencias responden a aspectos específicos de la formación sociopolítica. El ciudadano, es el primero de los conceptos que tiene una connotación política tanto en su orientación de lo político como de la política.

En el contexto global de los conceptos observados, "Ciudadanía" y su variante "ciudadano" y "ciudadana" y, siguiendo la propuesta de Lizcano (2012), es el primero en cantidad de frecuencias.

Según la localización del concepto, "Ciudadanía", es el tercero en frecuencias en los capítulos introductorios y uno de los más altos en la materia de "Educación para la Ciudadanía", mientras no tiene un lugar destacado dentro de las Ciencias Sociales. Esto nos lleva a intuir que tiene un papel importante en los aspectos declarativos del currículum y en la materia en sí, pero escaso en el ámbito temático al que corresponde que son las Ciencias Sociales.

Tabla 2

Frecuencias del concepto "Ciudadanía" según apartados del currículum

Apartados del currículum

Frecuencia

\begin{tabular}{llc} 
Capítulos & Competencias básicas & 20 \\
introductorios & Consideraciones generales de la etapa & 7 \\
& & Estructura de la etapa \\
& Total & 29 \\
\cline { 2 - 3 } Materias & Materia Ciencias Sociales, Geografía e Historia. Introducción & 13 \\
& general & 22 \\
& Materia Educación para el Desarrollo Personal y la Ciudadanía. & 22 \\
& Introducción general & \\
& Tercero. Educación para la Ciudadanía y Derechos Humanos & 4 \\
& Cuarto. Educación Ético-cívica & 8
\end{tabular}


RISE - International Journal of Sociology of Education, 6(1) 119

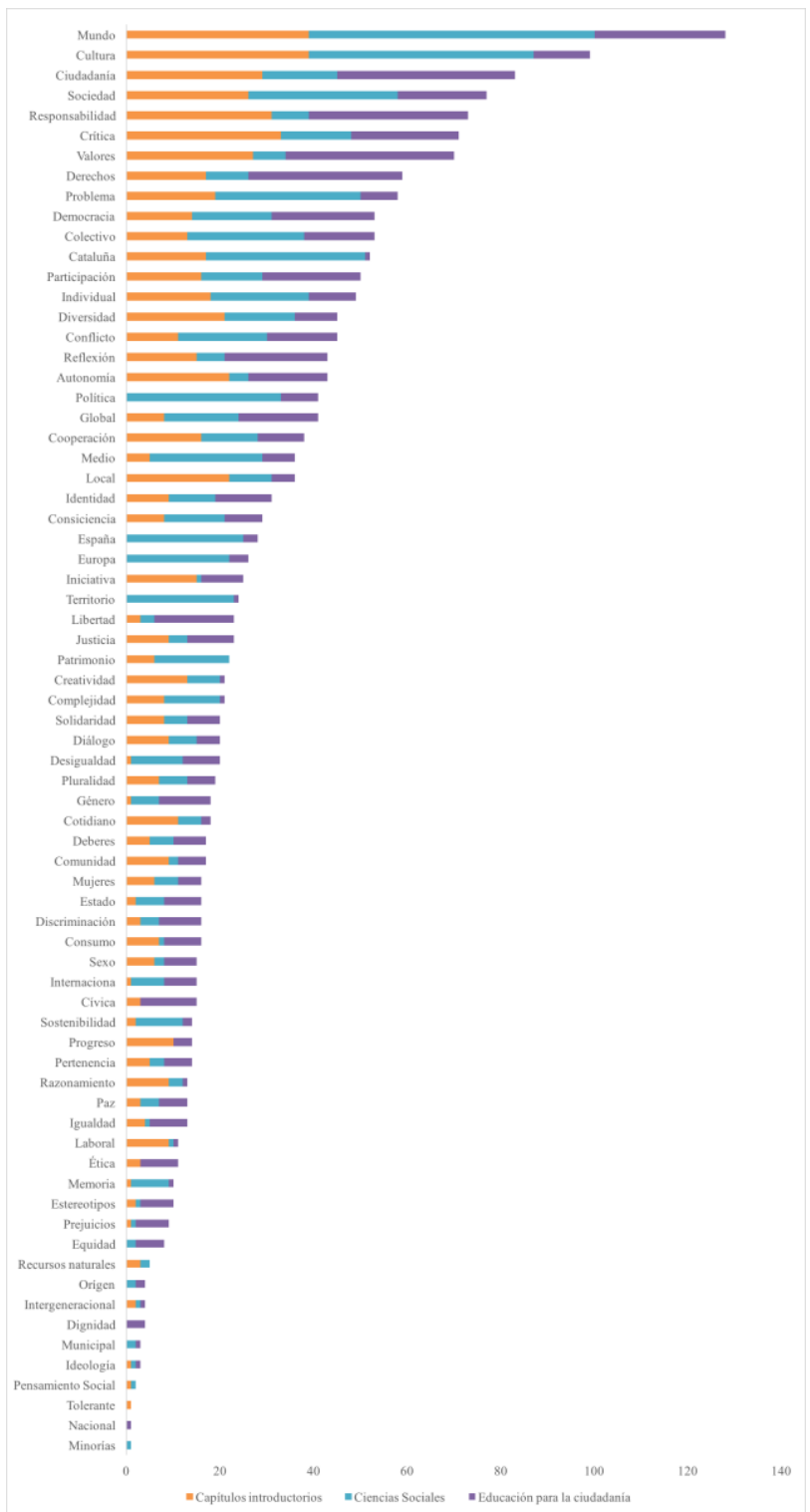

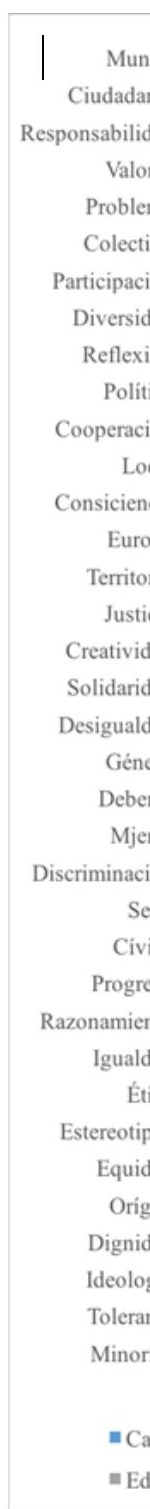

Gráfico 1. Frecuencias de conceptos sociopolíticos recogidos según apartados del currículum

Elaboración propia a partir del currículum 


\title{
120 Sáez-Rosenkranz, Bellatti \& Mayoral-Ciudadanía
}

Como se puede observar en la tabla 2, la frecuencia del concepto según apartados del currículum no es homogénea, en la parte general del currículum, aparece casi la mitad de las veces que en las materias específicas. Tiene una presencia muy superior en lo que corresponde a las competencias básicas en desmedro de otros sub apartados como la estructura o consideraciones generales de la etapa. Aquí la ciudadanía aparece como un ideal de formación, puesto que

\begin{abstract}
"además de desarrollar conocimientos, capacidades, habilidades y actitudes necesarias (el saber, saber hacer, saber ser y saber estar), los niños y niñas deben aprender a movilizar todos estos recursos personales (saber actuar) para conseguir la realización personal y convertirse en personas responsables, autónomas e integradas socialmente, para ejercer la ciudadanía activa" (Generalitat de Catalunya, 2007a, p. 18) ${ }^{4}$.
\end{abstract}

En el contexto específico de las materias, la ciudadanía adquiere una fisionomía distinta en "Ciencias Sociales, Geografía e Historia" y en "Educación para el Desarrollo Personal y la Ciudadanía”. En la asignatura de Ciencias Sociales, la frecuencia del concepto ciudadanía aparece alrededor de la mitad de las veces que en ciudadanía, y se utiliza para delimitar algunas las características de la formación en la asignatura. De esta manera plantea que "Las Ciencias Sociales, Geografía e Historia deben facilitar el desarrollo de la consciencia ciudadana del alumnado" (Generalitat de Catalunya, 2007a, p. 107), puesto que en "este mundo globalizado se exige la formación de un ciudadano con capacidad de análisis y razonamiento que aprenda a buscar, seleccionar y utilizar la información de manera sistemática y crítica [...]"' (Generalitat de Catalunya, 2007a, p. 108).

En el caso de la asignatura de Educación para la ciudadanía, las frecuencias más significativas aparecen la introducción general a la vez que también están presentes en las asignaturas específicas en tercero y cuarto. En la parte general, el concepto tiende a aparecer al igual que en los apartados anteriores como un aprendizaje en su educación, que implica el "desarrollo de ciudadanos y ciudadanas responsables y democráticos y posibilitar la transformación personal y la adquisición de competencias y habilidades necesarias que contribuyen al logro de una sociedad más libre, justa y 
equitativa" (Generalitat de Catalunya, 2007a, p. 142).

En el caso de las asignaturas específicas de esta materia, en tercer y en cuarto curso, aparece el concepto en el marco de contenidos específicos. Así por ejemplo, en tercer curso se indica que entre los contenidos se debe propender a la "valoración de las administraciones (local, estatal y europea) en la presentación de servicios públicos y de la importancia de la participación ciudadana en el funcionamiento de las instituciones" (Generalitat de Catalunya, 2007a, p. 149).

\section{Características del Concepto Ciudadanía a lo Largo del Currículum}

El concepto "ciudadanía" y sus palabras asociadas "ciudadano" y "ciudadana", aparece como adjetivo, es decir definiendo a un sustantivo y conformando un concepto, como por ejemplo "participación ciudadana". También se presenta como sustantivo, ya sea como un concepto en sí mismo ("ciudadana") o bien como parte de un concepto como en el caso de la "ciudadanía activa".

\section{Ciudadanía como Adjetivo}

Como adjetivo la ciudadanía define ciertos sustantivos y se localiza fundamentalmente en el cuerpo de las materias de "Ciencias Sociales, Geografía e Historia" y "Educación para el desarrollo Personal y la Ciudadanía". Allí aparece como definiendo la participación, la cooperación y la consciencia. El caso de participación ciudadana, es una participación relativa a la vida cívica (Generalitat de Catalunya, 2007a) para lo cual el currículum establece que se deben asumir valores democráticos y conocer la organización de los estados democráticos (Generalitat de Catalunya, 2007a). Esta participación planteada de forma general en los apartados introductorios, está circunscrita al funcionamiento y desarrollo de las instituciones democráticas en tercer curso y a las democracias actuales en el cuarto curso.

La precisión curricular de la existencia de una cooperación ciudadana, implica que se reconoce que existen diferentes tipos de cooperación, pero la que interesa para la formación de los estudiantes, es la ciudadana. Esta cooperación, se asocia a ciertas formas específicas que se materializan en el 


\section{Sáez-Rosenkranz, Bellatti \& Mayoral-Ciudadanía}

currículum en asociaciones y voluntarismo. La manera en las que el currículum presenta cooperación ciudadana, promueve su valoración por los estudiantes (Generalitat de Catalunya, 2007a), pero en ningún caso, propende a que sean criticadas, analizadas o complementadas con otras formas de cooperación.

En el caso de la conciencia ciudadana, aunque no se define ni contextualiza, se precisa que pretende "permitir al alumno dar sentido a las relaciones del pasado, el presente y el futuro, y su identidad territorial y cultural" (Generalitat de Catalunya, 2007a, p. 107).

\section{Ciudadanía como Sustantivo}

El concepto de ciudadanía como sustantivo se emplea tanto en los títulos como en el cuerpo de cada apartado, a diferencia de lo que ocurre como adjetivo, ya que allí no aparece encabezando ningún cuerpo de texto.

Los títulos cumplen la función de precisar qué tipo de educación se entrega, "educación para la ciudadanía y los derechos humanos" o "educación para el desarrollo personal y la ciudadanía", "pertenencia y ciudadanía", "competencia social y ciudadana". La ciudadanía aquí aparece como una característica propia, distinta de otros descriptores de la educación. Aunque el currículum no define explícitamente qué es una educación ciudadana, ni define ciudadanía queda implícito su individualidad respecto a otros conceptos como Derechos Humanos o la sociedad.

En este sentido se pueden plantear tres cosas. En primer lugar, que la formación ciudadana en la perspectiva curricular se relaciona con el desarrollo personal como condición para lo social. En segundo lugar, que la ciudadanía tiene que ver con la formación de la identidad personal y colectiva. Finalmente que la ciudadanía es concebida como una competencia. Esto lleva implícito una serie de conocimientos y habilidades que se deben poner en práctica ante determinadas situaciones (Perrenoud, 2006). Por tanto no se plantea necesariamente como una actitud de vida, sino como una aptitud que se pone en juego ante determinadas situaciones.

Dentro del cuerpo de los textos, la ciudadanía aparece como una condición diferente de los social y lo individual. Así se establece que "la educación escolar ha de prever situaciones problemáticas que todo ciudadano y ciudadana debe aprender a administrar y solucionar en los 
campos concretos de [...] la educación social y para la ciudadanía" (Generalitat de Catalunya, 2007a, p. 29). Es un aspecto relacionado directamente con una formación cívica y política, en tanto el estudiante debe "disponer de habilidades para participar activamente y plena en la vida cívica" (Generalitat de Catalunya, 2007a, p. 32). Este enfoque de la ciudadanía aparece más cercano a lo jurídico en desmedro de lo ético-cívico, puesto que como elemento jurídico-político, se centra en los derechos y deberes políticos, mientras que como formación ético-cívica se incorpora una idea de la vida pública con valores cívicos (Lizcano Fernández, 2012).

Como sustantivo, la ciudadanía también aparece formando parte de conceptos mayores: ciudadanía activa, ciudadanía democrática, ciudadanía responsable y comprometida y, finalmente ciudadanía informada y crítica. En estos casos, excepto en el de la ciudadanía democrática, la ciudadanía está asociada a acciones, por tanto, es un concepto orientado al movimiento. Ya no se presenta como un ideal en sí mismo, sino orientado a la capacidad del individuo de participar en su contexto social de forma consciente, de cara a la intervención en una organización política democrática. Esto es lo que Print denomina ciudadanía democrática (Print, 2003).

\section{Verbos y Conceptos Asociados a la Ciudadanía}

A pesar de que la palabra "ciudadanía" aparece numerosas veces a lo largo del currículum, los verbos y conceptos que la acompañan tienden a reiterarse a lo largo del texto. Las ideas que engloban estos conceptos pueden agruparse de la siguiente manera:

La ciudadanía como ámbito de la formación. El concepto educación aparece frecuentemente dentro de la idea de "educación para la ciudadanía" que pone de manifiesto la vinculación entre la formación del estudiante y la ciudadanía como elemento a aprender a lo largo de la etapa escolar. Como señala Cristian Cox y otros (2014), la ciudadanía no es inherente sino que se hace, es una construcción donde la educación y la escuela juega un papel central. De esta manera, plantearlo en términos curriculares, implica reconocer que el sistema educativo es uno de los encargados de desarrollar aprendizajes de cara al ejercicio de la ciudadanía.

Características de la ciudadanía. La ciudadanía aparece acompañada de 


\section{Sáez-Rosenkranz, Bellatti \& Mayoral-Ciudadanía}

conceptos que permiten caracterizarla. Aparecen ideas como la generación de un sentimiento de ciudadanía global; construcción de la paz y la democracia; actitudes y derechos y deberes de la ciudadanía. Estas nociones nos hablan de una ciudadanía que si bien no aparece definida en el currículum recoge algunos rasgos claros.

En primer lugar se vincula con un sistema político determinado y los atributos que cada sujeto tiene en dicho marco: la democracia y sus derechos y deberes. El currículum no precisa cuáles son esos derechos y deberes más allá de una referencia general a los derechos internacionales, al marco jurídico del Estado español o al de la Comunidad Autónoma de Cataluña.

En segundo lugar, la ciudadanía se asocia a una serie de valores detallados tanto en los objetivos como en los contenidos. El respeto a la diferencia, la responsabilidad, la solidaridad y, la valoración y cuidado del medio ambiente son las que aparecen claramente establecidas en el currículum. Destacamos aquí el tema de la diferencia, puesto que aparece en reiteradas ocasiones para el ámbito de género y diversidad social y cultural.

Para Chantal Mouffe (1999), los valores ético-políticos son fundamentales para el devenir de la democracia. Así, en el ciudadano convergen derechos, deberes y valores cívicos necesarios para su participación en la sociedad democrática (Print, 2003). De esta manera la definición explícita de estos elementos implica una cierta delimitación de la ciudadanía en el cual los derechos y deberes quedan al arbitrio de cada centro educativo -ya que el currículum no los precisa-, mientras que los valores y actitudes destacadas, constituyen los elementos mínimos que homogeneizan estos aprendizajes.

En tercer lugar, aparecen las dimensiones espaciales sobre las que opera la formación en el ámbito ciudadano. Aquí destacan lo global, lo europeo, estatal autonómico y lo local. Esto se relaciona directamente con las dimensiones individual y social que se promueve en el currículum en la formación ciudadana. Mouffe (1999) sostuvo que para participar políticamente, se requiere de una identidad política común que les permitan actuar gracias a la identificación con lo público.

Ciudadanía como acción. En el apartado prescriptivo del currículum de ESO son escasos los verbos que acompañan el concepto de ciudadanía, y sin duda 
el que más se repite a lo largo del documento es el "ejercer”. Su presencia está principalmente en infinitivo, pero también aparece conjugado o como sustantivo. Al tratarse de una palabra que apela al movimiento, se reconoce implícitamente que la ciudadanía es un aprendizaje que no solo se vincula con el conocimiento declarativo de cuestiones cívico políticas, sino que se prende sea puesto en práctica. Se declara en múltiples ocasiones la formación de una ciudadanía que permita ejercerla. Algunos investigadores sobre educación ciudadana sugieren sitúan a la ciudadanía como una praxis y señalan una serie de aprendizajes que en conjunto permiten desarrollarla y ejercerla (Print, 2003).

En términos discursivos, esta puesta en práctica de la ciudadanía se debe realizar según el currículum sobre la base de una serie de habilidades y aprendizajes que permitan actuar bajo este marco formativo. Algunas de estas acciones son el cooperar, valoración y conocimiento de la democracia y, la adquisición de normas de convivencia. Por tanto, la adquisición de la ciudadanía como aprendizaje para la vida, se centra en cuestiones que van más allá de la instrucción para entrar en el ámbito de la formación.

\section{¿Qué Deben Aprender los Estudiantes en Formación Ciudadana?}

La materia de Educación para el desarrollo personal y la ciudadanía, tiene por objetivo el desarrollo de tres grandes capacidades desglosadas en aspectos específicos. La primera capacidad, aprender a ser y actuar de forma autónoma, la segunda aprender a convivir y la tercera aprender a ser ciudadanos y ciudadanas en un mundo global.

Los verbos que guían estas capacidades son todos verbos que podríamos denominar activos, es decir que implican no solo la recepción de conocimientos, sino también la acción entorno a ellos. Sin embargo, al observar los descriptores para cada uno de ellos, es muy frecuente la presencia de verbos que podríamos denominar pasivos, es decir aquellos en los que los estudiantes adquieren un conocimiento o actitud, pero no se dirigen a la práctica. Así por ejemplo aparecen las ideas de "conocer y aceptar la propia identidad", "reconocer valores de esfuerzo personal", "conocer y asumir derechos y deberes", "identificar mecanismos de sociedades democráticas", "identificar los rasgos básicos del lenguaje de la 


\section{Sáez-Rosenkranz, Bellatti \& Mayoral-Ciudadanía}

publicidad", "reconocer el derecho de las mujeres" o "reconocer la diversidad social y cultural" entre otros enunciados (Generalitat de Catalunya, 2007a, pp. 146, 174). En todos estos casos, el papel del estudiante sugiere actitudes cognitivas que implican en última instancia el conocer, y escasamente poner en práctica ese conocimiento.

Sin embargo, hay otros enunciados de los descriptores de estas capacidades dirigidos a la acción, como por ejemplo el desarrollo de "la afectividad [...] rechazando prejuicios y estereotipos y las relaciones basadas en el dominio del otro", "Desarrollar la capacidad crítica y e iniciativa personal, asumiendo responsabilidades y actuando con autonomía" y "participar en actividades de grupo" (Generalitat de Catalunya, 2007a, pp. 146, 147). Mas, estos son menos frecuentes que los pasivos.

$\mathrm{Al}$ analizar los criterios de evaluación planteados para las asignaturas de tercer y cuarto curso, que por su carácter evaluativo deben dar cuenta del logro o no de los objetivos de la asignatura, se observa que están en la misma línea que las capacidades a desarrollar en la materia de forma más radical. En los dos cursos los verbos de evaluación son mayoritariamente pasivos como por ejemplo, "reconocer los componentes individuales y colectivos de la dimensión humana y expresar las propias emociones respetando las de otros desde la valoración de la dignidad y libertad humana" (Generalitat de Catalunya, 2007a, p. 150) o "valorar la dimensión libre y responsable de la acción humana, así como la dimensión moral de los comportamientos" (Generalitat de Catalunya, 2007a, p. 154).

Pero también hay algunos de carácter activo, aunque aparecen de forma secundaria o bien relacionados con procedimientos de contenidos conceptuales, como en el caso de "utilizar de forma rigurosa y contrastada diferentes fuentes de información para documentarse sobre algunos de los problemas y retos de la sociedad actual [...]" (Generalitat de Catalunya, 2007a, pp. 150, 155).

De esta manera, los aprendizajes que los estudiantes debiesen desarrollar a en la materia de formación ciudadana son la adquisición de una serie de contenidos y conductas que no necesariamente aspiran a que se pongan en práctica en el futuro, sino que en última instancia se quedan en la identificación, reflexión o valoración de un determinado tema o situación.

\section{Discusión y Conclusiones}


La ciudadanía es un aspecto clave en la formación de los estudiantes de ESO, aunque en el currículum el concepto aparece con mayores frecuencias como contenido específico en la asignatura de Formación Ciudadana, que como concepto trasversal en la enseñanza de las ciencias sociales. Este rasgo que fragmenta la formación en ciudadanía de la Historia, Geografía y Ciencias Sociales ha sido tratado en otras investigaciones (López Atxurra \& de la Caba Collado, 2012; Serna Dimas, 2015). Allí los autores señalan que existe un escaso vínculo entre ambos ámbitos formativos. Esta situación se tiende a corroborar con nuestros datos, los cuales sugieren que la ciudadanía no es un concepto clave en la enseñanza de las ciencias sociales. Sin embargo, esta última, es una temática idónea para trabajar temas ciudadanos (Prats, 2010), especialmente mediante la promoción del pensamiento histórico y el análisis social.

La tendencia a considerar la ciudadanía como un ámbito de aprendizaje independiente no sólo aparece en relación a la Historia y Ciencias Sociales, sino que cuando este concepto aparece dentro del texto como sustantivo, adquiere una fisionomía aparte de lo social y de lo individual. Implícitamente aquí se reconoce que no es una condición dada por la mera pertenencia a la comunidad, sino que la escuela es la encargada de desarrollarlas (Cox et al., 2014; Marri \& Keegan, 2014). De esta manera, la ciudadanía es un ámbito que involucra distintos aprendizajes, entre ellos los directamente cívicos y los de comportamiento en el orden social (Kerr, 1999).

Más, la forma en la que aparece este concepto en el currículum, se vincula con mayor intensidad a su dimensión social mediante la definición de conceptos como participación cooperación o la construcción de aprendizajes como pertenencia ciudadana. La vertiente individual también aparece asociada a la ciudadanía en lo que corresponde a la formación de conciencia de los individuos.

La literatura sugiere que la formación ciudadana debe ser promovida mediante el empleo de estrategias didácticas que la promuevan (Eurydice, 2006; Print, 2003). Sin embargo, al analizar los verbos de aprendizaje propuestos por el currículum, que guiarán la práctica docente, se desprende que tal meta se logra parcialmente. La gran mayoría de ellos remite a actitudes pasivas del alumnado cuyo producto se centra en la comprensión 


\section{Sáez-Rosenkranz, Bellatti \& Mayoral-Ciudadanía}

que este tenga de los aprendizajes ciudadanos y en menor medida aparecen aquellos en los que la dimensión ciudadana se pone en juego. Así, la formación del sujeto como un ser activo dentro de la sociedad, se ve truncado.

Nuestros resultados sugieren que en el currículum catalán la ciudadanía tiende a acercarse más hacia una acepción en términos jurídicos más que éticos, lo que de alguna manera reproduce la forma en la cual se ha trabajado el tema a lo largo de su inclusión en la escuela (Cristol et al., 2010). Por tanto, las perspectivas contemporáneas para el trabajo de la formación ciudadana en la escuela siguen siendo un campo aun por profundizar. En este sentido, tal como indica Morrone (2015), es necesaria una adaptación del concepto ciudadanía en educación a las nuevas exigencias de convivencia que demandan los colectivos sociales.

\section{Notas}

1Este artículo se ha llevado a cabo dentro de los siguientes proyectos: grupo de investigación consolidado DHIGECS (Didáctica de la Historia, la Geografía y otras Ciencias Sociales), financiado por la Generalidad de Catalunya (2014SGR-955); proyecto Recercaixa 2012 «Educación cívica en les aulas interculturales: análisis de las representaciones e ideas sociales del alumnado y propuestas de acción educativa» (2012ACUP00185), financiado por la Obra Social "la Caixa"; proyecto «Desarrollo de la formación sociopolítica para una ciudadanía democrática: diseño e implementación de materiales didácticos en Ciencias Sociales» (EDU2015-65621-C3-3-R), financiado por el Ministerio de Economía y Competitividad del Gobierno de España

2 Si bien desde 2014 ha entrado en vigor la Reforma Educativa de la Ley Orgánica para la Mejora de la Calidad Educativa (LOMCE) del Real Decreto 1105/2014, el presente curso escolar 2016/2017 sigue siendo un año de transición entre el actual y el pasado marco jurídico (LOE) . La LOMCE, aprobada en diciembre de 2013, se ha puesto en marcha de forma escalonada y entramos en el tercer curso de implantación, donde tiene que aplicarse en $2^{\circ}$ y $4^{\circ}$ de la ESO y en $2^{\circ}$ de Bachillerato, que hasta ahora se regían por el sistema anterior.

3 Debe decir: Un detalle más profundo de la metodología se puede ver en el texto "Anàlisi del currículum de Ciències Socials i Educació per a la Ciutadania en l'Ensenyança Secundària Obligatòria com a eina per a l'ús crític i participatiu de la professió docent" (Bellatti \& SáezRosenkranz, 2013)

4 Las citas textuales del currículum en catalán se han traducido y transcrito al castellano por los autores. 


\section{Referencias}

Arendt, H. (1997). ¿Qué es política? Barcelona: Paidós.

Coll, C. (1987). Psicología y currículum. Barcelona: Laia.

Cox, C., Bascopé, M., Castillo, J. C., Miranda, D., \& Bonhomme, M. (2014). Educación ciudadana en América Latina: Prioridades de los currículos escolares. IBE Working Papers on Curriculum, 14, 1-43.

Cristol, D., Michell, R., \& Gimbert, B. (2010). Citizenship Education: A Historical Perspective (1951-Present). Action in Teacher Education, 32(4), 61-69. doi: 10.1080/01626620.2010.549725

Eurydice. (2006). La educación para la ciudadanía en el contexto escolar europeo. Madrid; Luxemburgo: Ministerio de Educación y Ciencia, Subdirección General de Información y Publicaciones ; OPOCE.

Generalitat de Catalunya. (2007a). Currículum Educació Secundària Obligatòria.

Generalitat de Catalunya. (2007b). Decret 147/2007.

Gimeno Sacristán, J. (1988). El currículum: una reflexión sobre la práctica. Madrid: Morata.

Kerr, D. (1999). Citizenship education in the curriculum: An international review. School Field, 10(3/4), 5-32.

Kries, S. (2006). La ciudadanía. Una mirada histórica. En Discursos y prácticas de ciudadanía. Debates desde la Región de Bío Bío (pp. 19-27). Hualpén: Ediciones Universidad del Bío Bío.

Krippendorff, K. (1990). Metodología de análisis de contenido. Teoría y práctica. Barcelona: Paidós.

Lizcano Fernández, F. (2012). Conceptos de ciudadano, ciudadanía y civismo. Polis. Revista Latinoamericana, 32, 1-24.

López Atxurra, R., \& de la Caba Collado, M. Á. (2012). Educación para la participación ciudadana en los libros de texto: oportunidades y resistencias. En N. De Alba Fernández, F. F. García Pérez, \& A. Santisteban Fernández (Eds.), Educar para la participación ciudadana en la enseñanza de las Ciencias Sociales (Vols. 1-I, pp. 73-82). Sevilla: Asociación Universitaria de Profesorado de Didáctica de las Ciencias Sociales-Diada editora.

López Facal, R., \& Valls Montés, R. (2012). La necesidad cívica de saber história y geografía. En N. de Alba Fernández, F. F. García Pérez, \& 
A. Santisteban (Eds.), Educar para la participación ciudadana en la enseñanza de las ciencias sociales (pp. 185-192). Madrid; Montequinto, Sevilla: Asociación Universitaria de Profesorado de Didáctica de las Ciencias Sociales ; Díada.

López Noguero, F. (2002). El análisis de contenido como método de investigación. XXI. Revista de Educación, (4), 167-179.

Marri, A. R., \& Keegan, P. (2014). Developing Democratic Citizenship Among Youth. Theory \& Research in Social Education, 42(1), 147150. doi: 10.1080/00933104.2014.876893

Minte, A., Orellana, C., \& Tello, D. (2013). Formación Ciudadana en los textos escolares de Historia de Chile. Íber. Didáctica de las Ciencias Sociales, Geografía e Historia, 75, 18-24.

Morrone, A. (2015). Le forme della cittadinanza. En B. Borghi, F. García Fernández, \& O. Moreno Fernández (Eds.), Novi Cives. Cittadini Dall'infanzia in poi (pp. 3-14). Bolonia: Pàtron.

Mouffe, C. (1999). El retorno de lo político. Comunidad, ciudadanía, pluralismo, democracia radical. Barcelona: Paidós.

Perrenoud, P. (2006). Construir competencias desde la escuela. Santiago: J. C. Sáez Editor.

Piñuel Raigada, J. L. (2002). Epistemología, metodología y técnicas de análisis de contenido. Estudios de Sociolingüística, 3(1), 1-42.

Prats, J. (2010). En defensa de la Historia como materia educativa. Tejuelo, 9, 8-18.

Print, M. (2003). Estrategias de enseñanza para la educación cívica y ciudadana en el siglo XXI. ESE, 4, 7-22.

Bellatti, I., \& Sáez-Rosenkranz, I. (2013). Anàlisi del currículum de Ciències Socials i Educació per a la Ciutadania en l'Ensenyança Secundària Obligatòria com a eina per a l'ús crític i participatiu de la professió docent. En Una mirada al pasado y un proyecto de futuro. Investigación e innovación en didáctica de las ciencias sociales (Vol. 1, pp. 227-238). Barcelona: Servei de Publicacions Universitat Autónoma de Barcelona.

Serna Dimas, A. (2015). Ciencias sociales, pensamiento histórico y ciudadanía: entre lo alegórico y lo virtual. Revista de Estudios Sociales No.35, 52, 147-157. doi:10.7440/res52.2015.10

Stenhouse, L. (1997). Cultura y educación. Sevilla: Morón. 
Torres Santomé, J. (1991). El currículum oculto. Madrid: Ediciones Morata.

Isidora Sáez-Rosenkranz is Professor at the University of Barcelona, Spain

Ilaria Bellatti is Professor at the University of Barcelona, Spain

Dolors Mayoral is Professor at the University of Lleida, Spain

Contact Address: isidora.saez@ub.edu 